\title{
Alex Demirovic
}

\section{Balkankrieg, Postfordismus und Global Governance}

\author{
Ich will nicht in den Krieg. \\ Ich bin nicht auf der Welt, \\ um arme Leute umzubringen. \\ Boris Vian
}

Niemand wird mit realistischen Gründen annehmen können, daß die Grünen mit ihrer Beteiligung an der Regierungsverantwortung auch unmittelbar die Kompetenz gehabt hätten, über die Bombardements der NATO in Jugoslawien zu entscheiden. Die Kräfteverhältnisse in der Bundesregierung und in der NATO ließen dies nicht zu. Daß sie sich diesen Machtverhältnissen allerdings nicht entgegengestellt, sondern sie selbst mitvollzogen haben, verändert tiefgreifend die politische Konstellation. Die politische Handlungsweise der rot-grünen Regierung berührt nämlich den grundsätzlicheren Gesichtspunkt der Möglickeit zur Herstellung eines sozial-ökologischen New Deal.

In der Strukturkrise des Übergangs von der fordistischen zur postfordistischen Form der kapitalistischen Vergesellschaftungs steht eine Regulationsform zur Disposition, die auf Massenproduktion, hohem und wachsendem Einsatz von Ressourcen mit weitläufigen Belastungen der Gesellschaft wie der Natur und zunehmender Bürokratisierung in den Unternehmen und in öffentlichen Einrichtungen mit von oben geplanten und kontrollierten Formen der rigiden Abpressung von Mehrarbeit beruhte. Im Anschluß an regulationstheoretische
Überlegungen bestand die Überzeugung, daß ein neues Muster der Reproduktion und Regulierung des Kapitalverhältnisses nicht einfach linear aus dem Bisherigen folgen würde, sondern sich aus den sozialen Kämpfen zwischen Kapitaleignern, Gewerkschaften, sozialen Bewegungen, Parteien und vielfältigen Initiativen der Zivilgesellschaft gleichsam als ein Fundstück, als ein neuer Kompromiß herausbilden würde. Ein Kompromiß kann nur gelingen, wenn die unterschiedlichen Konfliktparteien um ihre eigenen und die Ziele der anderen wissen, ebenso aber auch wissen, daß sie jeweils ihre eigenen Positionen nicht als solche durchsetzen können. Dieser neue, sozial-ökologische New Deal würde zwar das Kapitalverhältnis selbst nicht beseitigen, aber doch wesentliche Strukturmomente verändern, nämlich die energetische Grundlage von fossilen und nuklearen auf erneuerbare Energieträger umstellen, den Ressourceneinsatz verringern, Bürokratisierung abbauen, das WarfareRegime durch Abrüstung in ein ökologisches Welfare-Regime verändern und offenere und durchlässige Lebensformen begünstigen.

Die Grünen repräsentierten die zahlreichen und diversen Initiativen und Grup- 
pen, die sich aus dem früheren, fordistischen Klassenkompromiß des nationalen, keynesianischen Wohlfahrtsstaat herauslösen wollten. Die Partei faßte diese teilweise sehr partikularen Interessen zusammen, verallgemeinerte sie und machte sie dadurch auch in der offiziellen Politik zu einem die politische Agenda bestimmenden Machtfaktor. Die Grünen stellten aufgrund dieser Entstehung und Funktion selbst einen Kompromiß dar, der nun von den Grünen in der Bundesregierung auf die Probe gestellt und mit der Entscheidung, die Bombardements der NATO zu unterstützen und die rot-grüne Koalition nicht $\mathrm{zu}$ verlassen, schließlich gebrochen wurde. Der Kompromiß umfaßte zahlreiche politische Issues, im Zusammenhang mit dem Balkankrieg sind insbesondere die drei folgenden von Bedeutung: a) die Ökologie; b) der Multikulturalismus; c) der Pazifizismus. In allen drei Hinsichten handelt es sich um eine Art Hegemonie des grünen Projekts über die Linke, denn die Linke der siebziger Jahre wurde damit integriert, ohne daß ihre kapitalismuskritischen Ziele in diesen Begriffen einen angemessenen Ausdruck fanden. $\mathrm{Zu}$ a) Der Begriff der Ökologie hat eine biologistische Färbung und reduziert die Komplexität des gesellschaftlichen Naturverhältnisses auf bloße Fragen der natürlichen Umwelt. Die Atomenergie war ein Schlüsselthema der Umweltproblematik, die Konzentration darauf stellt aber gleichfalls eine Reduktion dar. Die gegenwärtigen Verhandlungen der Bundesregierung mit der Atomindustrie legen nahe, daß auch weiterhin lange Laufzeiten akzeptiert werden, gleichzeitig scheint die Bundesregierung bereit, weitere Kernkraftwerke in der Ukraine zu finanzieren. $\mathrm{Zu} \mathrm{b}$ ) Der
Multikulturalismus tendiert dazu, die Vielfalt sozialer Verhältnisse, die mit der Migration verbunden sind und die massive Repression und Ausbeutung umfassen, auf Fragen der bloß kulturellen Anerkennung von nationalen bzw. ethnischen Unterschieden $\mathrm{zu}$ reduzieren. Damit werden künstliche Kollektividentitäten kreiert, die die Grundlage für rassistische Affekte bilden. $\mathrm{Zu}$ c) Der generelle, im Prinzip vor allem gegen die NATO und den Warschauer Block und die atomare Bewaffnung gerichtete Pazifismus ließ völlig offen, wie mit anderen als exterministischen Kriegen umzugehen sei. Schon früh ließ sich bei manchen grünen Realpolitikern ein instrumenteller Menschenrechtsmoralismus beobachten, wenn sie von der CDU-FDP-Regierung forderten, die Sandinisten wegen ihrer Verletzungen der Rechte der nicaraguensischen Ostküsten-Indianer wirtschaftlich zu boykottieren, die Menschenrechtsverletzungen der Contra und der bürgerlichen Rechten in den zentral- und lateinamerikanischen Ländern aber gar nicht beachteten. Schon bald nach der Wende 1989, und verbunden mit den Diskussionen über den zweiten Golfkrieg 1991, wurde unter Grünen mit Erleichterung festgestellt, daß nun endlich der Krieg wieder ein politisches Instrument sei und nicht jedesmal mit der atomaren Vernichtung gerechnet werden müsse. Menschenrechtlicher, politischer Pazifismus scheint die Formel für diese Haltung zu sein, die beansprucht, gegen naive Pazifisten verantwortungsethisch die Bürde $\mathrm{zu}$ tragen, eine neue Form des Gewaltmonopols zu schaffen, das weltpolizeilich gegen die kleinen Diktatoren durchgesetzt werden muß und Menschenrechte und Frieden erzwingt. Der usurpatorische, antidemokratische 
Charakter der Errichtung dieses Gewaltmonopols bleibt außer Betracht, vielmehr wird positiv noch unterstrichen, daß damit die universalistischen Normen des Westens durchgesetzt werden, wie sie von den USA beansprucht und vertreten werden. Die undemokratischen, rassistischen, gewaltsamen und ausbeuterischen sozialen Verhältnisse in den USA werden ebensowenig thematisiert wie die Bereitschaft der führenden kapitalistischen Staaten, die weltweiten, sich immer noch weiter vertiefenden wirtschaftlichen und politischen Asymmetrien zu erhalten. Die moralische Unentschiedenheit läßt sich für die gegenwärtige Kriegskampagne der NATO auch daran erkennen, daß die Türkei an der NATO-Kriegsführung mitwirkt, obwohl evident ist, daß sie selbst entschieden gegen die Prinzipien verstößt, auf denen die Operationen beruhen sollen: auf Zypern trennt türkisches Militär Griechen von Türken nach nationalistischen Gesichtspunkten; in der Türkei selbst wird den Kurden jedes Recht auf Autonomie verweigert.

Der Balkankrieg vertieft die moralischen Dilemmata, vor die sich die Grünen und die Linke schon während des Golfkrieges gestellt sah. Denn anders als während des damaligen Krieges ist Deutschland unmittelbar als Kriegspartei beteiligt und wird von einer rotgrünen Koalition regiert; die Entscheidungen werden von sozialdemokratisch-grünen Regierungen getroffen und von einem NATO-Generalseketrär ausgeführt, der sich selbst einmal als Pazifist verstand. Zwar widersprechen die militärischen Operationen der NATO dem Grundgesetz, dem NATO-Vertrag und haben kein UN-Mandat - das auch seinerzeit stark umstritten war. Entscheidend für das moralische Dilemma erscheint aber, daß dieses Mal nichts anderes als moralische Normen selbst zur Kriegsführung zu nötigen scheinen. Auf den ersten Blick sind keine geostrategischen Interessen im Spiel. Doch der Schein trügt.

Die gegenwärtige Kriegsführung steht in der Logik der deutschen Außenpolitik gegenüber Jugoslawien seit Beginn der neunziger Jahre. Während der serbische Nationalismus von deutscher Seite kritisiert und abgelehnt wurde, wurde der Nationalismus Kroatiens, wurden dessen Bestrebungen zur staatlichen Unabhängigkeit anerkannt. Damit wurde faktisch ein Regime unterstützt, das ständig Menschenrechte, zivile und politische Rechte verletzt, demokratische Freiheiten behindert und vor allem der Bereicherung weniger Familien und Warlords dient, die durch Nationalismus und Rassismus ihre Macht aus der früheren, staatssozialistischen Phase in kapitalistische Formen der Macht und des Reichtum zu konvertieren in der Lage waren. Die deutsche Außenpolitik ergriff im Namen von Demokratie und Selbstbestimmung faktisch Partei für einen Nationalismus gegen einen anderen Nationalismus und beteiligte sich damit an der Zerstörung des früheren Jugoslawien als der wahrscheinlich einzigen Form, die unterschiedlichen religiösen, regionalen und sogenannten ethnischen Widersprüche und Differenzen wenn nicht zu lösen, so doch auszubalancieren. Die demokratische Opposition, die sich in den früheren Teilrepubliken Kroatien, Bosnien und Serbien gebildet hatte, wurde nie ernsthaft unterstützt. Möglicherweise, weil sie vielfach eine Opposition war, die für die Erhaltung der Einheit des Landes und für eine Beseitigung der materiellen Privilegierung einzelner Volksgruppen 
eintrat. Dies war das Naheliegende, denn für viele Jugoslawen waren die Bürgerkriegserfahrungen während des Zweiten Weltkriegs durch die Erfahrungen überlagert, daß die verschiedenen Volksgruppen seit Jahrzehnten zusammenlebten, sich räumlich, familiär, beruflich, im Militär, im Studium und in der Migration mischten. Es waren die politischen Eliten, die vom westlichen Ausland Unterstützung erfuhren, die allenfalls noch schwach vorhandenen ethnisch-religiösen Spaltungslinien zu intensivieren. Fragt man sich nach den Motiven, so kann vermutet werden, daß die deutsche Außenpolitik eine Zersplitterung begünstigte, um die Möglichkeit der Herausbildung einer starken politischen Kraft in Osteuropa zu verhindern und insbesondere den Einfluß Rußlands zu schwächen. Doch richtete sich diese Politik auch gegen einen Rest an moralischer Autorität Jugoslawiens, die sich daraus ergab, das es sich der Logik des Kalten Krieges entzogen und ein eigenes Modell der Arbeiterdemokratie zu realisieren versucht hatte, das lange Zeit nicht nur unter europäischen Linken auf Interesse stieß, sondern auch ein erhebliches Ansehen unter den Ländern des Südens genoß und das Land zu einem der führenden Repräsentanten der blockfreien Länder machte. Im Prozeß der Restrukturierung Europas als einem einheitlichen Block innerhalb des globalen Wettbewerbs besteht offensichtlich die Bestrebung zur Vereinheitlichung. Eigenständige Entwicklungspfade werden im europäischen Haus oder vor der Tür der Europäischen Union nicht geduldet. Milosevic ist das vom Prozeß der europäischen Integration erzeugte Monster. Er betreibt für Serbien, was bei Tudjman geduldet wurde. Die mehreren hunderttausend serbischen Vertriebenen, die als Flüchtlinge in Jugoslawien leben, sind ebensowenig Gegenstand der menschenrechtlichen Sorge wie der Terror von kroatischen Banden gegen Muslime. Ebensowenig wird bei der gegenwärtigen Kriegskampagne auf dem Balkan bedacht, welche Konsequenzen sich für die zehntausenden Albaner in Serbien ergeben, von denen viele mit Serben verheiratet und befreundet sind; ebensowenig werden die nachhaltigen destabilisierenden Folgen für Bosnien und Mazedonien und die dortigen Versuche gesehen, die nationalistische und rassistische Logik einzudämmen.

Für die rot-grüne Regierung hat dieser Hintergrund eine fatale Konsequenz. Denn indem sie vorgibt, die Rechte einer Volksgruppe, der Kosovaren zu schützen und der militärischen Option zustimmt, bekräftigt sie einmal mehr die Logik der ethnischen Trennung. Die militärische Aktion einer auswärtigen Macht in einem Bürgerkieg zwischen der Zentralregierung und einer sezessionistischen Bewegung mußte zwangsläufig das befördern, was nun feststeht: daß nämlich Serben und Kosovaren sich als feindliche Völker wahrnehmen, die nicht mehr miteinander zusammenleben können. Die Förderung und Anerkennung der UCK durch die deutsche Außenpolitik (und durch den BND) stärkten diese Dynamik noch. Für die Konservativen und die Rechte in Europa - und dies läßt sich an einer Bundestagsrede des außenpolitischen Sprechers der CDU-Fraktion Lammers ablesen bestätigen die Vorgänge im Kosovo, daß multikulturelle politische Gebilde nicht lebensfähig sind, weil sie letztlich zum Bürgerkrieg führen werden. Dem Ziel, in der Bundesrepublik und in der Europäischen Union den Rassismus und 
ethnische Spaltungslinien abzubauen, wird mit der verfolgten Politik langfristig Schaden zugefügt.

Es gibt zahlreiche Anhaltspunkte dafür, daß die Bundeswehr und die NATO von langer Hand eine Ausdehnung ihres Operationsgebietes und eine Erweiterung ihrer Aufgaben betreiben. Out-ofarea-Einsätze humanitärer und friedenserhaltender Art waren die Vorbereitungen für friedenserzwingende Einsätze. Volker Rühe 1992: »Ich bleibe dabei, daß wir auf Kampfeinsätze im Augenblick weder materiell noch psychologisch vorbereitet sind. [...] Es geht auch nicht nur darum, die Soldaten, sondern die ganze Gesellschaft auf diese neuen Aufgaben [weltweiter Kampfeinsätze; $\mathrm{AD}$ ] vorzubereiten. Bei Blauhelmeinsätzen ist das schon gelungen. [...] Auf Dauer ist eine Beschränkung auf Blauhelm-Einsätze nicht realistisch. [...] Das kann sich alles entwickeln.« (Der Spiegel 30/1992). Es handelt sich um eine langfristige Politik zur Neudefinition deutscher Sicherheitsinteressen, die im Zuge des Globalisierungsprozesses weltumspannend gedacht werden. Die neue Strategie zeichnet sich seit dem Balkankrieg und der 50 Jahr Feier der NATO deutlicher ab. Die NATO behält sich gegenüber den UN und dem Sicherheitsrat das Recht auf die Kriegsführung vor und nimmt damit die klassischen Rechte des staatlichen Souveräns wahr. Dies soll nur für den Ausnahmefall gelten, heißt es abschwächend. Faktisch begünstigt dies noch entschiedener die Souveränitätsrechte der NATO, denn über den Ausnahmefall entscheidet selbst wiederum niemand sonst als die Mitglieder der NATO und dort diejenigen, die mächtig sind und über den Militärapparat verfügen. Insofern es sich bei der NATO um eine militärische Organisation handelt, wird der Ausnahmefall immer eher nach militärischen, weniger nach politischen Gesichtspunkten definiert, wie dies bei Entscheidungen der UNO zwangsläufig der Fall wäre. Weltweit wird den Regierungen gezeigt, daß die NATO beansprucht zu bestimmen, welche Umstände einen Staat zu einem Schurkenstaat machen und was ihm passieren wird. Daraus entsteht die Gefahr weiterer militärischer Eskalation. Denn autoritäre Regime werden auf ABC-Waffen zurückgreifen, um das Risiko der militärischen Intervention zu senken.

Der neuen Konzeption der NATO nach wird eine Sicherheitszone definiert, die Europa und den Raum des südlichen Mittelmeers umfaßt. Aber auch die kaukasischen und transkaukasischen Staaten - schon Kriegsziele der Wehrmacht - sind eingeschlossen. Gerade dort werden große und noch weitgehend unerschlossene Erdölvorkommen vermutet. Das unmittelbar von der NATO kontrollierte Gebiet wird auf diese Weise bis an die chinesische Grenze reichen. Sollte dieses Szenario zutreffen, dann schließt dies zweierlei ein. Die Bundesrepublik Deutschland beteiligt sich an einer langfristig angelegten Strategie zur Sicherung fossiler Energie. Die Gefahr besteht, daß die Entwicklung eines nachhaltig-zukunftsfähigen Energieregimes hinausgeschoben wird. Eine Reihe von Ländern der OPEC wird marginalisiert; ebenso wird massiv in die klassische Einflußsphäre Rußlands eingegriffen. Eine neue Hochrüstungspolitik - wie sie jetzt schon von Scharping angedeutet wird ist $\mathrm{zu}$ erwarten. Militärische Konflikte mit China oder Indien sind nicht auszuschließen. Diese langfristige Strategie 
der NATO widerspricht den Zielen der Linken und der Grünen, soweit letztere dafür stehen, die Ziele der neuen sozialen Bewegungen in die Parlamente und Regierungen einzubringen.

Nicht, daß die Grünen an politischer Herrschaft partizipieren und Kompromiße eingehen, soll hier kritisiert werden. Zu kritisieren ist, daß sie durch ihr politisches Handeln gerade die Bedingungen für einen sozial-ökologischen Kompromiß verschlechtern, insofern sie zentrale Ziele aufgeben und einen Paradigmawechsel in der Form der Ausübung der politischen Herrschaft mittragen.

Politische Herrschaft wird nicht mehr nationalstaatlich oder imperial ausgeübt, sondern in dem weit verzweigten Netzwerk von Elementen eines internationalen Staates. Als Knotenpunkte in diesem Netzwerk lassen sich die Nationalstaaten, die UNO und der Sicherheitsrat, die UN-Unterorgansisationen und die von der UN organisierte Konferenzen, die Weltbank und der Internationale Währungsfond, die EU mit ihren Apparaten, die NATO, internationale Regime, Gerichtshöfe usw. charakterisieren. Der Politikprozeß - ohnehin weitreichend dominiert von den global operierenden Unternehmen und den Akteuren auf den Finanzmärkten - verläuft wenig transparent, die Entscheidungsverfahren sind kaum formalisiert, so daß sich starke Interessengruppen oder Staaten und Staatengruppen durchsetzen können. In diesem Rahmen wurde das Konzept der global governance in die Diskussion gebracht, um die netzwerkartigen Politikverflechtungen gleichsam zu öffnen und der öffentlichen Diskussion und der Demokratisierung zugänglich zu machen. Als Mittel dazu werden allgemein seit der Welt- umweltkonferenz in Rio die Entfaltung einer innerstaatlichen, internationalen und globalen Zivilgesellschaft ebenso wie die Menschenrechte betrachtet. Üblicherweise meint Zivilgesellschaft einen zivilen, friedlichen, deliberativen Entscheidungsfindungs- und Interessenausgleichsprozeß. Doch ist daran zu erinnern, daß schon Gramsci, auf den das Konzept der Zivilgesellschaft (società civile) zurückgeht, auf den aggressiven, durchaus militärischen Aspekt der Zivilgesellschaft hingewiesen hat, der den demokratischen ergänzt. Gramsci entwickelte den Begriff der Zivilgesellschaft, um eine neue Form bürgerlicher Herrschaft $\mathrm{zu}$ charakterisieren. Mit Zivilgesellschaft wollte er auf die vielfältigen privaten Initiativen hinweisen, mit denen das Bürgertum nach dem Ersten Weltkrieg in Italien, bedroht durch die Rätebewegung, für die Aufrechterhaltung seiner Herrschaft kämpfte (zum Beispiel durch Schaffung von bewaffneten Banden). Aus der internationalen Zivilgesellschaft entwickelt sich auch heute eine Initiative und Bereitschaft zur Anwendung von Gewalt. Dies läßt sich an den vielfachen Plädoyers von Schriftstellern für die Bombardements der NATO feststellen. Dabei handelt es sich vordergründig nicht um militaristische Argumente. Allerdings wird dem moralischen Argument eine Bedeutung verliehen, die es plausibel erscheinen läßt, $\mathrm{daß}$ in seinem Namen getötet wird, während das im Namen von Interessen nicht als legitim gilt. So kann eine Abspaltung der materiellen Interessen der führenden NATO-Staaten von der von ihnen in Anspruch genommenen Moral vorgenommen werden. Die Hoffnung soll darin liegen, daß gleichsam hinter dem Rücken der Politiker und Militärs 
der NATO, die alle den Krieg nicht wollen - eine Weisheit, die sich schon bei Clausewitz ausgeführt findet: der Krieg dient immer dem Frieden - sich die Vernunft durchsetzt und zu einem weiteren Verrechtlichungsschub beiträgt, mit dem die Gewalt auf einem höheren Niveau wieder zivilisiert wird. Die Bombardements seien allein dann moralisch gerechtfertigt, wenn sie eng mit dem Ziel der Weltbürgergesellschaft verbunden bleiben. Wie so oft heiligt auch hier der Zweck die Mittel und wo gehobelt wird, fallen Späne. Aber ist es menschenrechtlich zu rechtfertigen, wenn den Vertriebenen und Flüchtlingen schließlich doch nicht geholfen wird und die serbische Bevölkerung aufs Schlimmste terrorisiert wird und ihre Lebensperspektiven verliert? Die Intellektuellen, die Akteure der Zivilgesellschaft, treten in ein neues Verhältnis zu Herrschaft und Macht ein und versuchen, die Entscheidungsträger dadurch zu lenken, daß sie ihnen die richtigen, menschenrechtlichen Formeln ansinnen. Auf diese Weise glauben sie, immer noch kritische Subjekte zu sein, die den politischen Prozeß auf Distanz abwägend zu beurteilen vermögen.

Doch die innere Zerrissenheit der Intellektuellen, gegen den interessengeleiteten, aber für den moralischen Krieg zu sein, trifft sich mit der zerrissenen Haltung der Politiker, die ihre Kriegsführungspolitik als Ergebnis eines moralischen Dilemmas präsentieren und damit eine neue Form der moderierenden Regierungskunst ausarbeiten: man will den Krieg nicht, aber man muß ihn führen, weil er das kleinere von zwei Übeln ist. Nüchtern und hart wird wie so oft eben für die Seite der Macht entschieden, die sich seit Jimmy Carter und der Trilateralen Kommission die
Form des Eintretens für die Menschenrechte gibt. In Deutschland führt die Inanspruchnahme der Menschenrechte zu einer Neubestimmung der internationalen Position und zu einer entschiedenen Moralisierung der Politik, die einhergeht mit einer Umschreibung der Geschichte. Auch wenn die für die Kriegsführung verantwortlichen Politiker schnell wieder davon abgekommen sind, die Situation im Kosovo mit den Deportationen während des Zweiten Weltkriegs und mit Auschwitz gleichzusetzen, so wird das Argument in einer schwächeren Version durchaus aufrechterhalten: Deutschland müsse die Freiheit, die es selbst erhalten habe, an andere weitergeben und dafür im Zweifelsfalle auch militärisch einstehen. Der deutsche Sonderweg - eine historisch immer fragwürdige Konstruktion - gilt nun als beendet. Dies ist gar nicht falsch. Ergab sich der deutsche Expansionismus aus der Konkurrenz zu den großen Kolonialmächten Frankreich und England, so hat Deutschland dazu gelernt und nimmt nun teil an einer neuen Form von Hegemonialpolitik, die sich weltweit Märkte, Arbeitskräfte und Ressourcen unterwirft. Indem innenpolitisch die Schwelle zur Kriegsführung gesenkt wird - was wahrscheinlich kaum eine andere als die rot-grüne $\mathrm{Re}$ gierung moralisch so glaubwürdig bewirken kann -, wird das ökonomisch starke Deutschland quasi automatisch in kurzer Zeit neben und in Kooperation mit den USA zur zweitstärksten Militärmacht der Erde.

Die Formel, aus Auschwitz gelernt zu haben und nun anderen Völkern die Freiheit zu bringen, ist revisionistisch, denn am Ende gibt sie dem rassistischen Massenmord doch noch den guten Sinn, daß Deutschland die Rolle des 
Mahners und Erziehers mit militärischen Mitteln einnehmen darf, weil es aus seiner Geschichte gelernt habe. Dies erlaubt schließlich die historische Verdrehung, daß sich Deutschland gerade gegenüber einem Land als Freiheitsverkünder aufspielt, das es schon einmal mit expansionistischen und rassistischen Motiven terrorisiert hat und dessen Bevölkerung den vielleicht entschiedensten Widerstand gegen die deutsche Besatzung leistete. Das Ziel der Wehrmacht war es, den Lebensraum im Osten von Juden und Slawen freizumachen. Der Antislawismus und der allgemeine Rassismus ist in der Bundesrepublik kaum einmal zum Gegenstand der sogenannten Aufarbeitung der Vergangenheit geworden.

In England knüpft New Labour zur Rechtfertigung des Krieges an den kolonialistischen Diskurs von der Bürde des weißen Mannes an. So wie damals Zivilisation werden heute Freiheit und Menschenrechte verbreitet. In Deutschland wird analog dazu die Formel verwendet, aus den eigenen Verbrechen gelernt zu haben. Die hegemoniale Anmaßung ist dieselbe. Denn weltweit soll den Menschen vorgeschrieben werden, wie sie freiheitlich zusammenleben sollen. Keine Zweifel, das Leben unter dem autoritär-nationalistischen Regime Milosevic' ist nicht frei. Aber auch die über den Militärapparat der NATO verfügenden Machtgruppen werden kaum zulassen, daß es zu selbstbestimmten Formen des Zusammenlebens kommt. Im Namen von Freiheit und Demokratie haben die USA Diktaturen wie die von Pinochet unterstützt, sie haben Pol Pot, die Taliban und Saddam Hussein finanziert und ausgerüstet; und sie verfechten eine weltweite Liberalisierung, die die Mehrheit der Menschen ihrer Freiheit und ihrer Menschenrechte auf Ernährung, Bildung und Lebenschancen beraubt oder $\mathrm{zu}$ berauben droht. Jugoslawien zerstören, es neu aufbauen und seine Bevölkerung umerziehen - wenn es Daniel Goldhagen sagt, klingt es vielleicht nicht so wie bei Pol Pot, präsentiert aber das deutsche Modell auf absurde Weise erfolgreich, nämlich so, wie es die Rechte immer gesehen hat. Daß jedoch die innere Demokratisierung Deutschlands gegen den hinhaltenden Widerstand von in den antikommunistischen Konsens integrierten gesellschaftlichen Gruppen durchgesetzt werden mußte und nationalistische und rassistische Traditionen bis auf den heutigen Tag fortleben, die nicht zuletzt froh sind darüber, nun an der Seite der ehemaligen Feinde zu kämpfen, bleibt dabei außer acht.

Die Gefahr der von der rot-grünen Regierung verfolgten Kriegspolitik besteht darin, daß sie die Rationalitätsansprüche in der deutschen Gesellschaft deutlich senkt. Dies beginnt mit den Uneindeutigkeiten um die Verhandlungen in Rambouillet. Denn es drängt sich der Eindruck auf, daß hier die jugoslawische Regierung systematisch in die $\mathrm{Si}$ tuation gebracht wurde, ihre Unterschrift nicht geben zu können unter einen Vertrag, der faktisch zur Besetzung des Landes durch die NATO und einer Abtrennung des Kosovo geführt hätte. Die Begründungen der Bombardements erweisen sich als fadenscheinig, wenn behauptet wird, es gehe um den Schutz von Menschen und die Angriffe richteten sich gegen Milosevic und seinen Herrschaftsapparat - während doch gleichzeitig zahlreiche zivile Opfer zu beklagen sind, die Infrastruktur Jugoslawiens zerstört wird und erhebliche ökologische Verwüstungen in Kauf ge- 
nommen werden. Es handelt sich um ein atavistisches, tribalistisches Kriegsmodell, die das ganze, völkisch aufgefasste Volk der »Serben« mit seinem Herrscher identifiziert und entsprechend straft. Durch die Zerstörung des Landes und der Menschen will man diesen treffen und zum Einlenken zwingen. Seine Souveränität bleibt gleichsam gewahrt, die Menschen hingegen werden traumatisiert und zerstört. Die Personalisierung und Ethnisierung des Feindbildes läßt die Ablehnung des Nationalismus durch serbische Oppositionelle ebensowenig mehr erkennen wie die Tatsache, daß es auch in Serbien einen Unterschied zwischen Herrschenden und Beherrschten gibt. Die Zerstörung der Infrastruktur trifft die materiellen Lebensbedingungen von einfachen Menschen, von Arbeiterinnen, Kranken, Kindern.

Ein Moment der neuen Regierungskunst ist die Selbstpräsentation der Politiker, die sich in einer tragischen Situation sehen, Krieg führen $\mathrm{zu}$ müssen, um den Völkermord zu verhindern. Der Hintergrund der neuen sozialen Bewegungen und die Medien erzeugen den Effekt einer intimen Nähe zum Außenminister und den Appell: Stell' Dir vor, Du wärst an Joschkas Stelle - wie würdest denn Du handeln? Es wird eine Virtuosität der Rollenübernahme und des Perspektivenwechsels unterstellt und abgesehen von der Macht einer solchen politischen Position, die eben nicht von denen eingenommen werden könnte, die nicht so zu handeln bereit wären.

Auch die propagandistische Rhetorik und Manipulation verringert die Rationalität. In der Presse wird alles mögliche Kritische gesagt, aber gleichsam zusammenhang- und folgenlos. Die Re- gierung handelt jenseits der Öffentlichkeit; nicht einmal, daß Krieg ist, nicht einmal, daß die NATO sich zukünftig das Recht auf Selbstmandatierung, also das Ius belli, vorbehält, mag sie zugeben. Während in den Analysen des Bundesverteidigungsministeriums nüchtern von serbischen Polizeiaktionen gegen UCK-Kämpfer gesprochen wird, stellt Minister Scharping dieselben Vorgänge der Presse als Massaker dar. Es ist eine Gewöhnung ans Unlogische: jede Untat der serbischen Polizei und des Militärs, die doch durch den Krieg eigentlich hätte verhindert werden sollen, wird zum Anlaß weiterer und noch schärferer Bombardements. Das Motiv der Bestrafung und Rache dominiert allmählich über die menschenrechtlichen Argumente: die Serben sollen spüren, was den Kosovaren angetan wird - so als seien serbische Menschen weniger schützenswert. Die rot-grüne Regierung verwendet das Instrument des Krieges eben nicht rational und realpolitisch, sondern moralisch und überläßt sich deswegen seiner Logik, die eben die ist, die Feindschaft zu intensivieren.

Die neue Regierungskunst zielt darauf, die deutsche Öffentlichkeit abzuschleifen und sie langfristig zu passivieren. Ähnlich wie in den USA wird es auch in Deutschland zu einer Professionalisierung des Krieges kommen; auch hier sprechen Soldaten mittlerweile davon, daß sie ihren Job gut erledigen. Das zivile und das militärische Leben werden parallel nebeneinander hergehen. Es wird nicht mehr der Krieg der großen Massen sein wie im Ersten und Zweiten Weltkrieg. Der Krieg als normalisierter technischer Beruf kann tagtäglich, wie jetzt schon mit den Bombardements im Irak, ausgeübt werden. 
Der Konflikt über den Balkankrieg ist letztlich ein Konflikt darüber, ob am Ende der Ritt auf dem Tiger NATO doch noch dem guten Zweck dient oder ob Anpassung und Unterwerfung droht, daß also den Kosovaren nicht geholfen ist, aber auch weiterreichende Ziele der Grünen und Linken Schaden genommen haben werden. Die Bilanz für die grünen und linken Kriegsbefürworter ist negativ. Die Grünen an der Regierung tragen nicht dazu dabei, die Arkana der Politik der öffentlichen Diskussion zugänglich zu machen. Die Manipulation der Öffentlichkeit wird im Gegenteil verstärkt fortgesetzt. Die Ziele der Linken und der Grünen werden nicht mehr verfolgt. Die politische Rationalität wird geschwächt. Wie in der SPD, so zeigt der Vorgang, werden rationale politische Diskussionen um Programme und Ziele um des bloßen Machterhalts und der Staatsraison entwertet. Dies gilt einseitig als Fähigkeit, politisch zu handeln. Die Entwicklung läßt ein postfordistisches Herrschaftsprojekt erkennen, an dem die Grünen zwar beteiligt sind, jedoch um den Preis, nicht mehr für einen sozial- ökologischen Kompromiß einzutreten. 